\title{
Real-world heart failure management in 10,910 patients with chronic heart failure in the Netherlands
}

\author{
Design and rationale of the Chronic Heart failure ESC guideline-based Cardiology practice \\ Quality project (CHECK-HF) registry
}

\author{
J. J. Brugts ${ }^{1}$ - G. C. M. Linssen ${ }^{2}$ A. W. Hoes ${ }^{3,4} \cdot$ H. P. Brunner-La Rocca ${ }^{5} \cdot$ CHECK-HF investigators \\ Published online: 21 March 2018 \\ (c) The Author(s) 2018. This article is an open access publication.
}

\begin{abstract}
Aims Data from patient registries give insight into the management of patients with heart failure (HF), but actual data from unselected real-world HF patients are scarce. Therefore, we performed a cross sectional study of current HF care in the period 2013-2016 among more than 10,000 unselected HF patients at HF outpatient clinics in the Netherlands.

Methods In 34 participating centres, all 10,910 patients with chronic HF treated at cardiology centres were included in the CHECK-HF registry. Of these, most (96\%) were managed at a specific HF outpatient clinic. Heart failure was typically diagnosed according to the ESC guidelines 2012, based on signs, symptoms and structural and/or functional cardiac abnormalities. Information on diagnostics, treatment and co-morbidities were recorded, with specific focus on drug therapy and devices. In our cohort, the mean age was 73 years (SD 12) and 60\% were male. Frequent co-morbidities reported in the patient records were diabetes mellitus 30\%, hypertension 43\%, COPD 19\%, and renal insufficiency $58 \%$. In $47 \%$ of the patients, ischaemia was the origin of HF. In our registry, the prevalence of HF with preserved ejection fraction was $21 \%$.

Conclusion The CHECK-HF registry will provide insight into the current, real world management of patient with chronic $\mathrm{HF}$, including HF with reduced ejection fraction, preserved ejection fraction and mid-range ejection fraction, that will help define ways to improve quality of care. Drug and device therapy and guideline adherence as well as interactions with age, gender and co-morbidities will receive specific attention.
\end{abstract}

Keywords Heart failure - Guideline · Adherence $\cdot$ Registry

\section{Introduction}

J. J. Brugts

j.brugts@erasmusmc.nl

1 Department of Cardiology, Erasmus Medical Center, Thoraxcenter, Rotterdam, The Netherlands

2 Department of Cardiology, Hospital Group Twente, Almelo and Hengelo, The Netherlands

3 Julius Center for Health Sciences and Primary Care, Utrecht, The Netherlands

4 University Medical Center Utrecht and Utrecht University, Utrecht, The Netherlands

5 Department of Cardiology, Maastricht University Medical Center, Maastricht, The Netherlands
Chronic heart failure (HF) is an important health care problem with high cardiovascular morbidity and mortality [1]. The prevalence of HF is $1-2 \%$ in developed countries and is expected to rise even further in the next decades [2]. Chronic HF has a disturbing prognosis if untreated or insufficiently treated, while suboptimal treatment is often not sufficiently recognised [3, 4].

We could improve the prognosis considerably when we would optimise drug and device therapy according to the European Society of Cardiology (ESC) guidelines [5], at least in the patients with $\mathrm{HF}$ and reduced left ventricular ejection fraction (HFrEF). Optimal treatment also includes adequate attention to lifestyle habits and management of co-morbidities. Considering medication, a guideline-based 
approach directly enhances the quality of care delivered to HF patients. However, older surveys such as the EuroHeart survey showed that only a minority of the patients were treated adequately with these agents, and when considering target dose the figures are even worse [6]. More recent surveys show much better adherence to HF treatment, but these surveys have shortcomings such as limited representativeness of included patients or lack of information on drug dose [7-9]. Furthermore, most studies did not distinguish between HFrEF and HF with preserved LVEF (HFpEF) [7-9]. We should consider these aspects if we want to assess adherence to guidelines in daily practice, which is a clear marker of quality of care delivered by health care professionals. Suboptimal adherence to guidelines is often claimed to be due to the presence of co-morbidities, but this has not been sufficiently studied. Therefore, it is important that we enhance awareness of the complexity of modern HF care, which includes the management of co-morbidities.

For better insight into these clinically relevant aspects, we set up a large cross-sectional study to assess current treatment of HF patients at outpatient clinics. The aim of the study was to describe clinical characteristics and treatment of unselected patients with HF in the Netherlands. Notably, we will evaluate how recommendations of the European guidelines regarding pharmacological and non-pharmacological treatments are adopted in clinical practice and to find determinants of non-adherence.

\section{The CHECK-HF study design}

The CHECK-HF (Chronisch Hartfalen ESC-richtlijn Cardiologische praktijk Kwaliteitsproject-HartFalen) is a Dutch registry aimed at improving quality of $\mathrm{HF}$ care in the Netherlands.

The current study is a cross-sectional registration of all unselected patients diagnosed with chronic HF who are treated at Dutch outpatient HF clinics (96\%) in the period September 2013-September 2016. All patients with HF seen at the general cardiology outpatient clinic of the same hospitals $(4 \%)$ could be included as well. There are no available data that allow us to estimate the exact proportion of patients from the centres that was included in our database. The objective was to include all enlisted patients. In view of the estimated average number of patients per centre the average proportion of included outpatient clinic patients with HF is well above $80 \%$. In total, 34 Dutch hospitals, including 1 academic hospital agreed to participate (Supplementary material list of participating centres and investigators) (Fig. 1).

The ethics committee approved our study (METC 2017 - Maastricht University Medical Center, Maastricht, the Netherlands).

\section{Type of patients}

Patient were included if the diagnosis of HF was based on the criteria of the ESC guidelines, that is signs and symptoms, and structural and/or functional cardiac abnormalities [5]. Heart failure was subtyped in HFrEF $(\mathrm{EF}<50 \%)$ or $\mathrm{HFpEF}(\mathrm{EF} \geq 50 \%)$ according to the guidelines that were in force at that time. Baseline ejection fraction assessments by echocardiography were available for the majority of patients at inclusion, while in some this was only classified semi-quantitatively (in a very small proportion, information was not provided). In the patients with available $\%$ ejection fraction, the whole spectrum of LVEF according to the new guidelines classification including the new group of mid-range EF (HFmrEF) with an EF of 40 to $49 \%$ can be studied [10]. No further exclusion criteria were applied apart from age $<18$ years. Aetiology of HF was further defined as ischaemic or non-ischaemic and further specified as hypertensive, valvular, related to arrhythmias or other, if applicable.

\section{Baseline characteristics and demographics}

The recorded dataset at inclusion consisted of age, sex, length, weight, body mass index, heart rate, systolic blood pressure, diastolic blood pressure, date of diagnosis, date of first outpatient visit, date of inclusion, New York Heart Association (NYHA) class, EF, HFrEF or HFpEF, pacemaker, implantable cardioverter defibrillator (ICD) or cardiac resynchronisation therapy (CRT), QRS duration, basic rhythm, laboratory values (serum creatinine, urea, potassium, sodium, brain natriuretic peptide (BNP), N-terminal pro hormone BNP (NT-proBNP)), co-morbidity and participation in cardiac rehabilitation programme.

\section{Assessment of current medication use}

The recorded dataset includes in-depth information (type, dosage and frequency, total daily dose) of the use of beta-blockers, angiotensin converting enzyme inhibitors (ACE) or angiotensin receptor II antagonists (ARB), mineralocorticoid receptor antagonists (MRA), ivabradine, diuretics (furosemide, bumetanide, hydrochlorothiazide, triamterene), digoxin, amiodarone, and statins. The use of platelet inhibitors and/or oral anticoagulants (yes/no) was recorded as well. 
Fig. 1 The 34 participating centres in the Netherlands

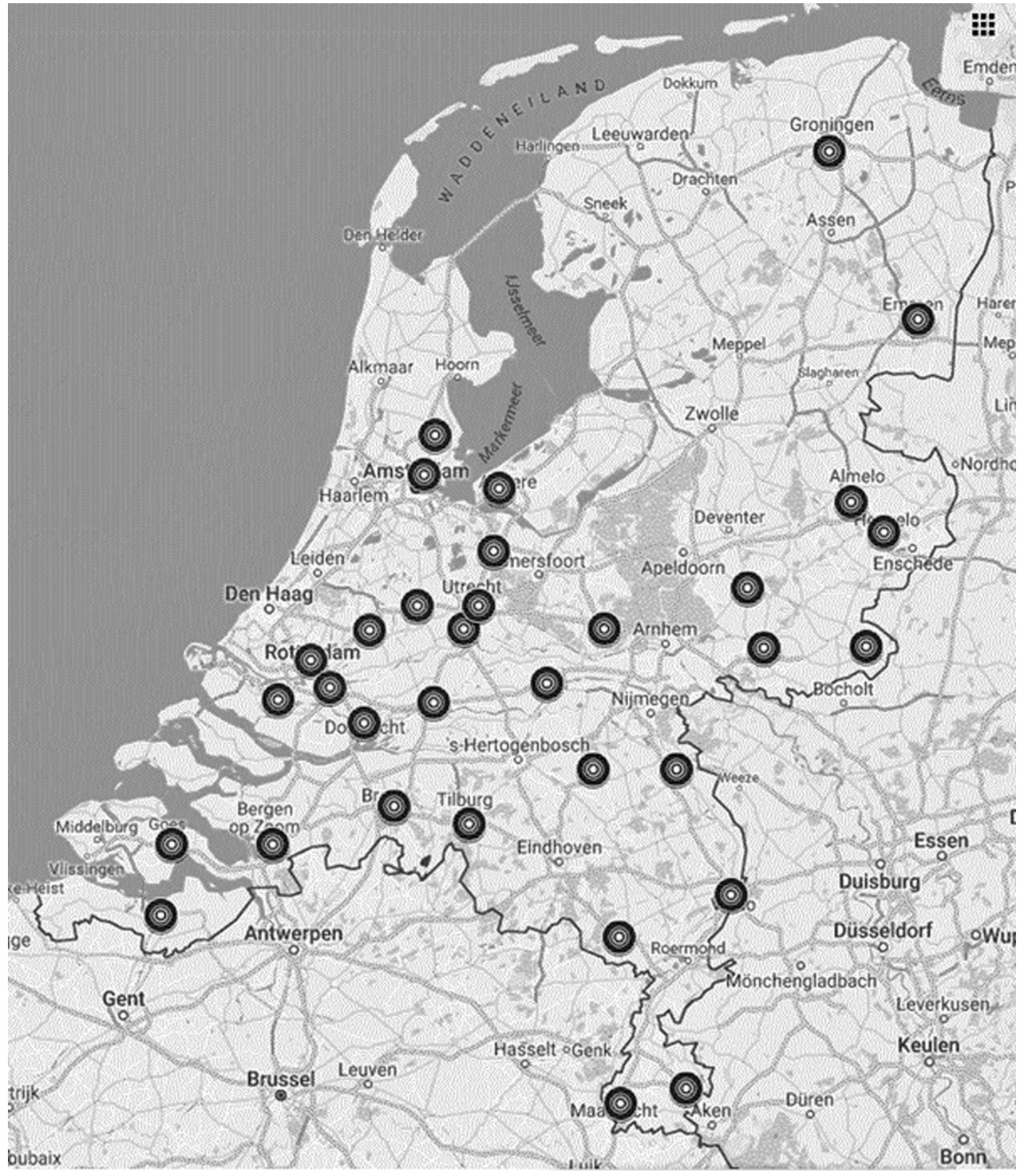

\section{Echocardiographic parameters}

At inclusion, we recorded values of the most recent echocardiogram of the patient with estimated ejection fraction in percentage either by biplane Simpson or Teicholtz, or left ventricular function semi-quantitatively assessed as normal function or mildly, moderately or severely impaired function. In the majority of patients, quantitative values of EF were available and recorded $(73 \%)$. Diastolic function, presence of restrictive filling pattern of the left ventricle as well as semi-quantitative assessment of inferior vena cava dimension/collapse, and pressure gradient across the tricuspid values were recorded.

\section{Co-morbidities}

The recorded dataset specifically noted the presence of co-morbidities as recorded in medical history (diabetes mellitus, hypertension, hypercholesterolaemia, renal insufficiency (estimated glomerular filtration rate $<60$ ), anaemia (haemoglobin below age-dependent threshold), thyroid dysfunction, chronic obstructive pulmonary disease (COPD) or obstructive sleep apnoea, and peripheral arterial occlusive disease).

\section{Data inclusion}

Data inclusion was performed using the CHECK-HF database based on Microsoft Excel with Visual Basic for Application designed to digitalise characteristics of 
Table 1 Baseline demographics in 10,910 patients with chronic heart failure (CHECK-HF)

\begin{tabular}{|c|c|}
\hline \multicolumn{2}{|l|}{ Baseline characteristics } \\
\hline Age, years (mean, SD); $n=10,890$ & $72.8 \pm 11.9$ \\
\hline Gender, $\%$ male; $n=10,859$ & $6523(60.1 \%)$ \\
\hline Body mass index, $\mathrm{kg} / \mathrm{m}^{2}$ (mean, SD); $n=9989$ & $27.5 \pm 5.4$ \\
\hline Hypertension, \%; $n=9733$ & $4173(42.9 \%)$ \\
\hline Diabetes mellitus, $\% ; n=9733$ & $2877(29.6 \%)$ \\
\hline Renal insufficiency ${ }^{\mathrm{a}}, \% ; n=8690$ & $5055(58.2 \%)$ \\
\hline COPD, $\% ; n=9733$ & $1829(18.8 \%)$ \\
\hline \multicolumn{2}{|l|}{ Aetiology: $n=10,468$} \\
\hline Ischaemic cardiomyopathy, $\%$ & $4877(46.6 \%)$ \\
\hline Non-ischaemic cardiomyopathy, $\%$ & $5591(53.4 \%)$ \\
\hline \multicolumn{2}{|l|}{ Heart failure: $n=10,627$} \\
\hline Reduced ejection fraction, $\%$ & $8360(78.7 \%)$ \\
\hline Preserved ejection fraction, $\%$ & $2267(21.3 \%)$ \\
\hline Unknown, \% & $283(2.6 \%)$ \\
\hline LVEF, \% (mean, SD); $n=9178$ & $36.0 \pm 14.1$ \\
\hline \multicolumn{2}{|l|}{ NYHA class; $n=10,664$} \\
\hline $\mathrm{I}, \%$ & $1808(17.0 \%)$ \\
\hline II, \% & $5884(55.2 \%)$ \\
\hline III, \% & $2777(26.0 \%)$ \\
\hline IV, $\%$ & $195(1.8 \%)$ \\
\hline Heart rate, bpm (mean, SD); $n=10,659$ & $72.2 \pm 14.1$ \\
\hline Systolic BP, mm Hg (mean, SD); $n=10,664$ & $127.6 \pm 21.5$ \\
\hline Heart failure outpatient clinic; $n=10,780$ & $10,385(96.3 \%)$ \\
\hline
\end{tabular}

$B P$ blood pressure, $b p m$ beats per minute, $C O P D$ chronic obstructive pulmonary disease, $e G F R$ estimated glomerular filtration rate, $H F$ heart failure, $L V E F$ left ventricular ejection fraction, MI myocardial infarction, NYHA New York Heart Association, $S D$ standard deviation

${ }^{a}$ Defined as eGFR below 60 or history of renal failure

patients and patient flow at the outpatient HF clinics. Each hospital recorded their data in a uniform stand-alone online database. Patient data were encoded and exported to a central database, fully anonymised, and cannot be traced to an individual patient. Data inclusion was based on a standardised protocol including a set of variables. As data collection was based on daily practice not all variables were available in all patients.

\section{Outcome measures}

The main analysis will present the adherence to guideline-directed medication-based therapy and device-based therapy in HFrEF patients. We will analyse adherence as percentage $(\%)$ of prescriptions as well as percentage (\%) of target dose of guideline-recommended heart failure treatments [5]. In addition, we will analyse the percentage of prescribed ICD therapy and CRT defibrillator (CRTD) therapy. Adherence data will be presented in future manuscripts. Secondary analyses will focus on specific subgroups of reduced and preserved left ventricular ejection fraction (LVEF) as well as comorbidities.

\section{Statistical analysis}

Summary statistics are provided as percentages (\%) or as mean with standard deviations (SD).

Baseline continuous variables are presented as mean $\pm \mathrm{SD}$ or median and interquartile range, depending on the distribution of the data; categorical data are presented as counts and percentages. We will compare the categorical variables using the $\chi^{2}$ test and the continuous variables using the $t$ test or the Mann-Whitney $U$-test, as appropriate. Univariate and multivariate regression analysis will be used to calculate odds ratio and 95\% confidence interval. Drug dose will be calculated compared with the recommended dose according to guidelines as percentage of actual recommended daily dose. Analyses are and will be performed with SPSS system software (version 24.0 or later).

\section{Results}

Demographics of the enrolled patients can be found in Tab. 1. About $60 \%$ were male and the average age was 73 years, with a wide range between 18 and 103 years of age (median 75 years, interquartile range (IQR) 66-82 years). Co-morbidities were common and in about half of the patients their HF was of ischaemic origin (46\%). The vast majority of patients had reduced left ventricular ejection fraction (79\%). Mean ejection fraction was 37\% (SD 14). Most patients were in functional NYHA class II (55\%).

\section{Discussion}

The current study will evaluate current quality of HF care in unselected patients with heart failure in the Netherlands. The CHECK-HF registry will demonstrate current adherence to ESC guidelines in the period of 2013-2016, that is between the publications of the 2012 and 2016 guidelines [5, 10]. Importantly, in CHECK-HF we not only collect data on presence or absence of medication, but also on the exact prescribed dosage. Knowledge regarding adherence to current recommended treatment, including dosage, is important as it can provide new insights on how we can improve current prescription rates and current practice.

The current study population of chronic HF patients in a real word setting differs from clinical trials by the high percentage of female patients and elderly patients and high prevalence of multiple co-morbidities. These 


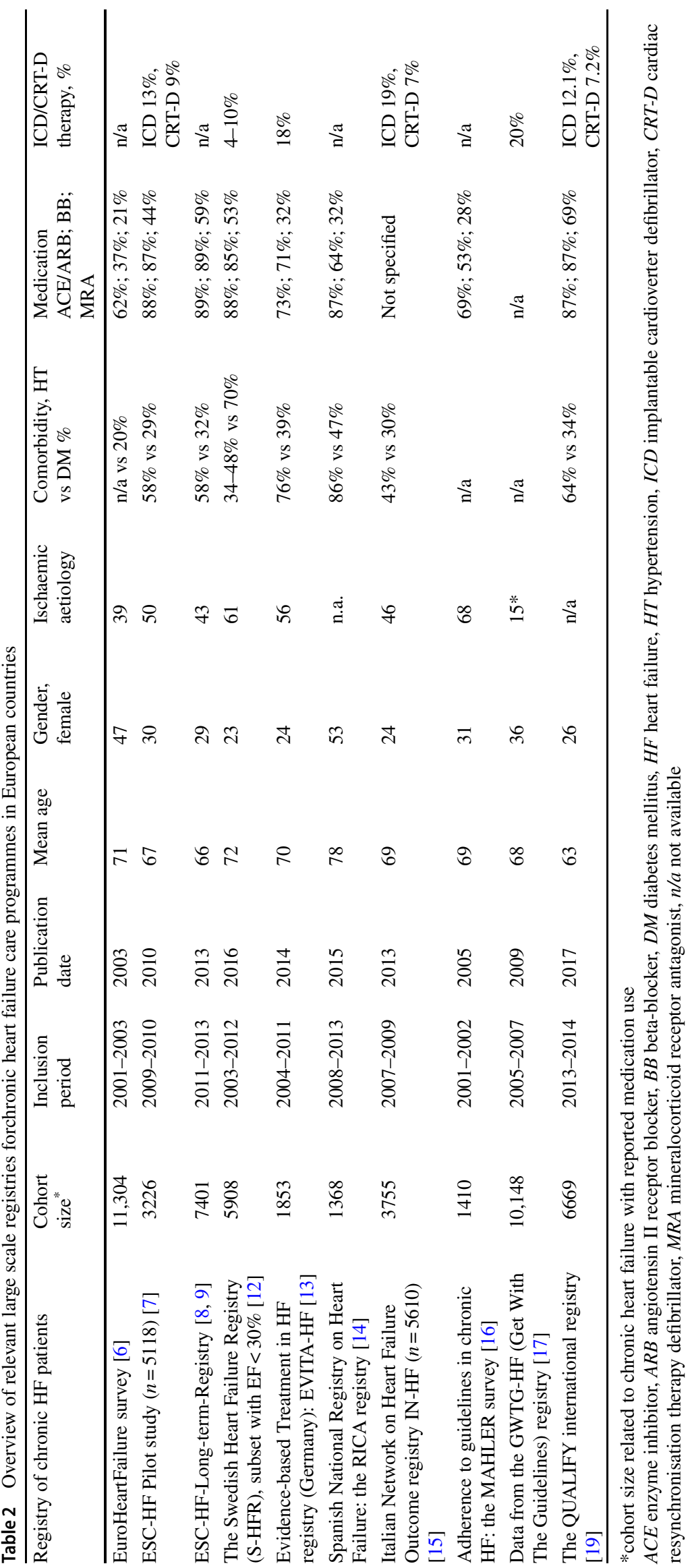


categories are chronically underrepresented in clinical trials, but form a significant part of the HF population seen in daily practice. The CHECK-HF registry will evaluate these subgroups with specific attention in secondary analyses studying treatment differences or differences in comorbidity distribution.

\section{The CHECK-HF registry}

The CHECK-HF registry consisted of a relatively high proportion of HFpEF patients $(21 \%)$. As noted in previous literature, there is a high percentage of female and/or elderly patients in this subgroup. The ESC guidelines had no specific treatment recommendations for HFpEF due to lack of evidence in randomised clinical trials. The CHECKHF registry will describe how these patients are treated and evaluate the distribution of age, gender and co-morbidity within this subgroup of HFpEF patients.

The number of co-morbid conditions in a patient with chronic HF is high and has been increasing in the last decade [11]. The majority of patients have 5 or more comorbid chronic conditions which are important determinants of the number of hospital re-admissions [11]. We can argue that in order to reduce the number of $\mathrm{HF}$ re-admissions, we should focus on influencing the level of comorbidities [11]. We can also hypothesise that earlier interventions could lower the extent of co-morbidities and collateral damage. There is a variety of examples of relatively simple solutions which may affect prognosis such as iron supplementation in iron deficient HF patients. Other examples are vitamin D deficiency, obstructive sleep apnoea syndrome and subclinical hypothyroidism. Secondary analyses of the CHECK-HF registry will focus on the level of co-morbidities in a real-world cohort and study potential interaction with the level of co-morbidities and treatment adherence. However, given the set-up of the CHECK-HF registry, we only have information for a selected number of co-morbidities.

\section{Comparison with other registries}

Several large national $\mathrm{HF}$ registries have been published in Europe [6-9, 12-17]. In Tab. 2 we provide an example of relevant major European $\mathrm{HF}$ registries with more than thousand patients with a report on heart failure medication and/or ICD therapy in specific patients with chronic heart failure [6-9, 12-17]. We particularly want to address two registries. The European Society of Cardiology Heart Failure (ESC-HF) pilot study was a prospective, multicentre, observational survey conducted in 136 cardiology centres from 12 European countries selected to represent the different health systems and care attitudes across Europe [7]. The ESC-HF pilot 2010 included 3,226 patients with chronic HF; mean age was 67 years and percentage of female patients was $29.7 \%$, both figures much lower than in the CHECK-HF registry and significantly lower than expected in a real-world HF population [7]. The adherence to recommended therapy in $\mathrm{HF}$ was $88 \%$ for ACE inhibitors or ARBs, $87 \%$ for beta-blockers, and $44 \%$ for MRA, but no distinction was made between patients with reduced versus preserved LVEF regarding therapy [7]. Moreover, there is limited available information on dosage, which will be an important strength of CHECK-HF. The ESC-HF long-term registry (ESC-HF-LT) was a prospective, observational registry in 211 cardiology centres from 21 European and/or Mediterranean countries, all being member countries of the ESC [8]. Between May 2011 and April 2013, it collected data on 12,440 patients, $40.5 \%$ of them hospitalised with acute HF and $59.5 \%$ outpatients with chronic HF. Thus, the ESC-HF-LT registry evaluated 7,401 patients with chronic HF with a mean age of 66 years and $28.8 \%$ female patients. Again, these figures are much lower compared with the current real world HF registry in CHECK-HF [8, 9]. The mean EF was 35\%. Overall adherence to $\mathrm{HF}$ medication was high with $89 \%$ for ACE inhibitors, $88 \%$ for beta-blockers, and $59 \%$ for MRA, but again no information on appropriateness of their use. The ARNO Observatory study by the Italian National Health Service (INHS) consisted of 41,413 patients who were discharged for HF from 1 January 2008-31 December 2012 and prescribed at least one HF treatment [18]. ACE inhibitors/ARBs, beta-blockers, and mineralocorticoid antagonists were prescribed in $65.8 \%, 49.7 \%$, and $42.1 \%$ of patients respectively. During 1-year follow-up, at least one rehospitalisation occurred in $56.6 \%$ of patients, $49 \%$ of them due to non-cardiovascular causes [18].

\section{Strengths of the CHECK-HF registry}

Overall, the CHECK-HF registry has several strengths. The CHECK-HF registry is one of the largest of its kind with almost 11,000 patients included. Moreover, it reflects actual real-world care as performed on outpatient HF clinics with a larger proportion of females and elderly when compared with other registries and clinical trials in particular, which better represents actual daily practice in many hospitals. The CHECK-HF registry has detailed information on medication use and dosage. The CHECK-HF registry also has information on co-morbidity levels and some biomarkers. Because there is extensive detailed information available, the CHECK-HF registry can provide more insight in relatively large subgroups of patients with $\mathrm{HFpEF}$ or HFmrEF and specific subsets of patients with atrial fibrillation and heart failure. A limitation of our study is the lack of detailed follow-up data. We plan to collect longitudinal data 
in the near future to report on the quality of HF care in the Netherlands and intend to perform several cross-sectional follow-ups of outpatient clinics in the Netherlands, with the perspective of repeated analyses of CHECK-HF as a longterm HF care research project. Longitudinal post-hoc data can potentially be obtained from mortality data from Dutch national archives (Statistics NL - CBS).

In conclusion, the CHECK-HF registry is a large HF registry which enrolled nearly 11,000 unselected patients with chronic HF treated at an outpatient clinic setting in the Netherlands to evaluate current HF management in 2013-2016 in a real-world setting. Specific attention will be given to gender and age differences and level of co-morbidities regarding HF treatment and guideline adherence in future analyses.

Complete list of co-authors/investigators of the CHECK-HF study H. van Amerongen, A. Derks, D. Hering, H.J. Kruik, M. Martherus, J. Pluimers, C.E.M. Rodijk-Heijmer, A. Uitzetter, D. Veldhuis (Hospital Group Twente Almelo/Hengelo); N.A.M. Huisman, A. van der Spank, J. Winter (Flevo Hospital Almere); A.H.M. Moons, M. Smit (MC Slotervaart Amsterdam); (Rijnstate Arnhem); R.M. Oortman (Bravis Hospital Bergen op Zoom); N. Aengenend, H.J.J. Koornstra-Wortel, T. Rongen (Maas Hospital Pantein Boxmeer); K.J. Balhuizen; J. Plomp (Tergooi Blaricum/Hilversum); A.A.M. van Drimmelen, I. Snoek (Amphia Hospital Breda); A. van Anken, L. van Rijn, F.J.J. Smeele (Slingeland Hospital Doetinchem); M.W.F. van Gent, G.C. van Lingen-Koppejan, P.A. Smits, H.I.S. Trossèl (Albert Schweitzer Hospital Dordrecht); H.J. Schaafsma, G. Tuin-v.d. Kolk, H.D. Vermeulen-v.d. Wetering, J. Zimmerman (Hospital Gelderse Vallei Ede); A. Adema, J.W. Brakel, M.J. Nagelsmit, W. Veenstra (Scheper Hospital Emmen); I. Aksoy, D.C. Meulmeester-Sinke, P.S. Monraats, H.H. Reijnierse-Buitenwerf, A.H. Witkam-Bal (Admiraal De Ruyter Hospital Goes); M. Boes-van Laar, H.M.C. SchoepBezemer, P.H.M. Westendorp (Rivas Beatrix Hospital Gorinchem); A. van Dieën, E.P. Viergever, E.B. Vossebelt (Groene Hart Hospital Gouda); L.H. Takens, W.E.H. de Valk-Bedijn (Martini Hospital Groningen); C.L.B. van der Bolt, R. Hendrick, J.A. Kragten, N.P. Stoot (Zuyderland Medisch Centrum Heerlen); M.A. Barandiaran Aizpurua, N.G.H.M. Marcks, J. Merken (Maastricht UMC Maastricht); L. Corsten, J.C. Kelder, R.M. van Tooren (St. Antonius Nieuwegein); T. Hillebrink, L. Oosterom, N. Telgt (Waterland Hospital Purmerend); B.M. van Dalen, A. van Miltenburg, N. Slingerland, B. Sonneveld (Franciscus Gasthuis Rotterdam); E. Bird-Lake, J. Hoek-Verschoor, A. van der Ree (Maasstad Hospital Rotterdam); A. Erol-Yilmaz, L. den Hartog-Taai, P. Middelburg-Poldervaart, (Spijkenisse Medisch Centrum Spijkenisse); P.C. Rademaker, S. de Smet, E.G.M.V. de Theije, T.J. de Wit (ZorgSaam Hospital Terneuzen); J. Langerveld, C.J. Morang-van Drempt, M.M. Vermeulen (Hospital Rivierenland Tiel); Y. Foolen, A.C.B. Pronk, B.M. Szabó, L.K. Valk (ETZ Elisabeth Tilburg); M.J.W. Grosfeld (Bernhoven Uden); M. Aertsen, D.J.M. Engelen, V. Kneijber, J. van Santvoord (Diakonessenhuis Utrecht); L.W.M. Eurlings, E.J. Geurts, R. Hazeleger, A.M. KoopmanVerhagen, G. Maessen, C.W.A.M. Pansters (VieCuri Medisch Centrum Venlo); P.R. Geerlings (St. Jans Gasthuis Weert); M. de Boer, A. Kolkman, C. van der Lee (Hospital Koningin Beatrix Winterswijk); R. Blonk, J. Krijger, J.P.P. Smits (Zuwe Hofpoort Hospital Woerden); N.Y.Y. Al-Windy, M. Harmsen (Gelre Hospitals Zutphen).

Acknowledgements We greatly acknowledge the participation of nurses and cardiologists of all participating sites who are specialised in the treatment of heart failure for including patients and storing patient data. We acknowledge the work of Rik van de Kamp (Servier Pharma, the Netherlands) for the development of the software programme. All authors contributed to the analysis of the data and writing of the report. All authors approved the final version of the manuscript.

Funding Servier, the Netherlands, funded the inclusion of data and software programme. The steering committee (JB, GL, AH, HBRLR) received no funding for this project. This combined analysis was initiated by the authors and was designed, conducted, interpreted, and reported independently of the sponsor. The current study had no other funding source or any with a participating role in outcome assessment, or writing of the manuscript. All authors had joint responsibility for the decision to submit for publication.

Conflict of interest J.J. Brugts, G.C.M. Linssen, A.W. Hoes and H.P. Brunner-La Rocca on behalf of CHECK-HF investigators declare that they have no competing interests.

Open Access This article is distributed under the terms of the Creative Commons Attribution 4.0 International License (http:// creativecommons.org/licenses/by/4.0/), which permits unrestricted use, distribution, and reproduction in any medium, provided you give appropriate credit to the original author(s) and the source, provide a link to the Creative Commons license, and indicate if changes were made.

\section{References}

1. Chioncel O, Lainscak M, Seferovic PM, et al. Epidemiology and one-year outcomes in patients with chronic heart failure and preserved, mid-range and reduced ejection fraction: an analysis of the ESC Heart Failure Long-Term Registry. Eur J Heart Fail. 2017; https://doi.org/10.1002/ejhf.813.

2. Schmidt M, Ulrichsen SP, Pedersen L, et al. Thirty-year trends in heart failure hospitalization and mortality rates and the prognostic impact of co-morbidity: a Danish nationwide cohort study. Eur J Heart Fail. 2016;18:490-9.

3. Stewart S, MacIntyre K, Hole DJ, et al. Five year survival following a first admission for heart failure: more malignant than cancer. Eur J Heart Fail. 2001;3:315-22.

4. Levy WC, Mozaffarian D, Linker DT, et al. The Seattle Heart Failure Model: prediction of survival in heart failure. Circulation. 2006;113:1424-33.

5. McMurray J, Adamopoulos S, Anker SD, et al. ESC guidelines for the diagnosis and treatment of acute and chronic heart failure. Eur J Heart Fail. 2012;14:803-69.

6. Komajda M, Follath F, Swedberg K, et al. The EuroHeart Failure Survey programme - a survey on the quality of care among patients with heart failure in Europe. Part 2: treatment. Eur Heart J. 2003;24:464-74.

7. Maggioni AP, Dahlstrom U, Filippatos G, et al. Heart Failure Association of the European Society of C. EURObservational Research Programme: regional differences and 1-year follow-up results of the Heart Failure Pilot Survey (ESC-HF Pilot). Eur J Heart Fail. 2013;15:808-17.

8. Maggioni AP, Anker SD, Dahlstrom U, et al. Are hospitalized or ambulatory patients with heart failure treated in accordance with European Society of Cardiology guidelines? Evidence from 12,440 patients of the ESC Heart Failure Long-Term Registry. Eur J Heart Fail. 2013;15:1173-84.

9. Crespo-Leiro MG, Anker SD, Maggioni AP, et al. European Society of Cardiology Heart Failure Long-Term Registry (ESC-HFLT): 1-year follow-up outcomes and differences across regions. Eur J Heart Fail. 2016;18:613-25. 
10. Ponikowski P, Voors AA, Anker SD, et al. ESC Guidelines for the diagnosis and treatment of acute and chronic heart failure: the task force for the diagnosis and treatment of acute and chronic heart failure of the European Society of Cardiology (ESC). Developed with the special contribution of the Heart Failure Association (HFA) of the ESC. Eur J Heart Fail. 2016;2016:891-975.

11. Braunstein JB, Anderson GF, Gerstenblith G, et al. Non-cardiac co-morbidities increases hospitalization and mortality among medicare beneficiaries with chronic HF. J Am Coll Cardiol. 2003;42:1226-33.

12. Thorvaldsen T, Benson L, Dahlström U, et al. Use of evidencebased therapy and survival in heart failure in Sweden 2003-2012. Eur J Heart Fail. 2016;18:503-11.

13. von Scheidt W, Zugck C, Pauschinger M, et al. Characteristics, management modalities and outcome in chronic systolic heart failure patients treated in tertiary care centers: results from the EVIdence based TreAtment in Heart Failure (EVITA-HF) registry. Clin Res Cardiol. 2014;103:1006-14.

14. RICA investigators, Montero-Perez-Barquero M, Manzano L, Formiga F, et al. Utility of the SENIORS elderly heart failure risk model applied to the RICA registry of acute heart failure. Int $\mathrm{J}$ Cardiol. 2015;182:449-53.
15. IN-HF (Italian Network on Heart Failure) Outcome Investigators, Tavazzi L, Senni M, Metra M, et al. Multicenter prospective observational study on acute and chronic heart failure: one-year followup results of IN-HF (Italian Network on Heart Failure) outcome registry. Circ Heart Fail. 2013;6:473-81.

16. Komajda M, Lapuerta P, Hermans N, et al. Adherence to guidelines is a predictor of outcome in chronic heart failure: the MAHLER survey. Eur Heart J. 2005;26:1653-9.

17. Shah B, Hernandez AF, Liang L, et al. Hospital variation and characteristics of implantable cardioverter-defibrillator use in patients with heart failure: data from the GWTG-HF (Get With The Guidelines-Heart Failure) registry. J Am Coll Cardiol. 2009;53:416-22.

18. Maggioni AP, Orso F, Calabria S, et al. The real-world evidence of heart failure: findings from 41413 patients of the ARNO database. Eur J Heart Fail. 2016;18:402-10.

19. Komajda M, Cowie MR, Tavazzi L, et al. Physicians' guideline adherence is associated with better prognosis in outpatients with heart failure with reduced ejection fraction: the QUALIFY international registry. Eur J Heart Fail. 20171;19:1414-23. 